\title{
The economic costs of diabetes in developing countries: some concerns and recommendations
}

\author{
A. K. Khuwaja • L. A. Khowaja • P. Cosgrove
}

Received: 16 September 2009 / Accepted: 6 October 2009 /Published online: 28 October 2009

(C) Springer-Verlag 2009

Keywords Developing countries $\cdot$ Diabetes $\cdot$ Economic cost

To the Editor: We read with great interest the recently published article by Esteghamati and colleagues [1] entitled 'The economic costs of diabetes: a population-based study in Tehran, Iran'. The authors are to be congratulated for their admirable work in measuring the economic burden of diabetes, a disease for which the number of people affected is growing rapidly in developing countries [2]. With reference to the article, we wish to comment on the growing burden of diabetes - especially in the context of developing countries - and then go on to make some recommendations.

It is estimated that the total number of people with diabetes globally will rise from 171 million in the year 2000 to more than 366 million in 2030 [2]. Diabetes is a complex metabolic disorder involving many body organs and systems and can devastate the lives of affected individuals. People with diabetes are up to four times more

\footnotetext{
A. K. Khuwaja $(\varangle)$

Department of Family Medicine, Aga Khan University,

Karachi 74880, Pakistan

e-mail: ali.khuwaja@aku.edu

L. A. Khowaja

Department of Community Health Sciences,

Aga Khan University,

Karachi, Pakistan

P. Cosgrove

Faculty of Social Sciences,

The Open University in the North West,

Sharston,

Manchester, UK
}

likely to develop cardiovascular diseases, and up to 40 times more likely to require lower limb amputation, than people without diabetes [3]. Furthermore, diabetes is among the leading causes of visual impairment, blindness and kidney failure in adults [3]. Diabetes is the fourth leading cause of death by diseases worldwide; more than one million people died from diabetes in 2005 and almost $80 \%$ of these deaths occurred in low- and middle-income countries [3, 4]. Due to the premature disability, morbidity and mortality associated with diabetes, diabetes is one of the most costly diseases to manage [5, 6]. This burden is even more of a problem in developing countries where diabetes and associated cardiovascular diseases develop at earlier ages and with greater severity [7]. The majority of people with diabetes in developing countries are below 64 years of age, whereas in developed countries most are in older age groups [2]. This early onset of diabetes in the more economically productive years of life leads to a devastating extra burden of cost in developing countries. This not only puts extra pressure on individuals, but affects families, society, and the healthcare system of the country.

Esteghamati and colleagues [1] determined the annual economic cost of individuals with diabetes in Iran. They reported significantly higher direct and indirect costs of diabetes compared with the control group. Moreover, the authors identified medications and devices as the most expensive component of direct costs. These results are consistent with other researchers' findings and estimates from developing countries [5, 6]. Nevertheless, we are concerned that these results may underestimate the economic cost of diabetes in Iran.

However, some of the methods used by Esteghamati and colleagues [1] are either imprecise or misleading. First, the control group includes people with hypertension and 
dyslipidaemia: these conditions have adverse cost and resource implications. In this way the authors have exaggerated the cost of the population group without diabetes, thereby reducing the comparative cost of people with diabetes. Second, the authors only estimated productivity losses for people with diabetes who are economically active; hence, it would appear that many people with diabetes have been excluded from the estimates-including housewives, students and retired people. Moreover, the age range of the study participants was from 0 to 104 years, so includes two extreme age groups comprising economically non-productive segments of the population. Yet no explanation is given as to how this aspect of the analysis was conducted. Third, patients' out-of-pocket expenses should be treated as direct costs, as with visits to healthcare providers [8]. However, while people with diabetes are likely to incur significant direct non-healthcare costs [5], in the study by Esteghamati and colleagues [1] this cost is treated as an indirect cost. Fourth, although healthcare in Iran is subsidised by the government [9], the estimation of healthcare costs in the study appears to have been based on insurance and out-of-pocket expenditures, with no adjustment indicated for any disparity between prices and the real costs of healthcare interventions. Finally, the loss of economic productivity from attendants accompanying patients is not calculated: many attendants will be economically active and so not taking their lost productivity into account may underestimate the economic cost of diabetes. If all these underestimated costs are taken into consideration, the actual economic cost will be much higher for people with diabetes relative to those without diabetes.

The existing scenario is one of a projected increase in the prevalence of diabetes for developing countries, where a large proportion of people are living below the poverty line and are dependent on fragile healthcare systems with limited resources. Prevention is the best option to tackle this costly epidemic. Preventive interventions should be available at all levels, with integrated, comprehensive, multi-faceted and multi-sectoral approaches. In this regard, activities should be aimed at preventing diabetes from occurring in susceptible individuals and populations through prevention and modification of environmental and behavioural risk factors and determinants. Because diabetes emerges rapidly in young people, special interventions should be planned and implemented at schools and colleges to target children and adolescents in developing countries. Activities such as early screening for diabetes (aiming at early detection), and prompt and effective management of the disease, should be designed with the purpose of preventing and delaying the onset of complications. Efforts should also be made to delay onset of the premature disabilities and the deaths associated with secondary complications of diabetes. Furthermore, improved standards of care and management of diabetes in the healthcare systems of developing countries would particularly help to ensure that all persons with diabetes have easier access to high-quality management of their disease at an affordable cost.

Duality of interest The authors declare that there is no duality of interest associated with this manuscript.

\section{References}

1. Esteghamati A, Khalilzadeh O, Anvari M et al (2009) The economic costs of diabetes: a population-based study in Tehran, Iran. Diabetologia 52:1520-1527

2. Wild S, Roglic G, Green A, Sicree R, King H (2004) Global prevalence of diabetes: estimates for the year 2000 and projections for 2030. Diabetes Care 27:1047-1053

3. International Diabetes Association (2003) Diabetes atlas, 2nd edn. International Diabetes Association, Brussels

4. World Health Organization (2008) Fact sheet no. 312. Diabetes. Available from www.who.int/mediacentre/factsheets/fs312/en/, accessed 31 August 2009

5. Khowaja LA, Khuwaja AK, Cosgrove P (2007) Cost of diabetes care in out-patient clinics of Karachi, Pakistan. BMC Health Serv Res 21:189

6. Ramachandran A, Ramachandran S, Snehalatha C et al (2007) Increasing expenditure on health care incurred by diabetic subjects in a developing country: a study from India. Diabetes Care 30:252-256

7. Khuwaja AK, Qureshi R, Fatmi Z (2007) Noncommunicable diseases and injuries: action needed in South Asia. PLoS Med 4: e38. doi:10.1371/journal.pmed.0040038

8. Elliot R, Payne K (2005) Essentials of economic evaluation in healthcare. Pharmaceutical Press, London

9. Kermani MS, Ghaderi H, Yousefi A (2008) Demand for medical care in the urban areas of Iran: an empirical investigation. Health Econ 17:849-862 